\title{
Effet du sel sur le comportement des jeunes plants de palmier à huile (Elaeis guineensis Jacq.) en Basse Casamance
}

\author{
Aichatou D. LABO ${ }^{*}$, Seyni SANE ${ }^{1}$, Daouda $\mathrm{NGOM}^{2}$ et Léonard E. AKPO ${ }^{1}$ \\ ${ }^{1}$ Laboratoire d'Ecologie et d'Agroforesterie, Département de Biologie Végétale, \\ Faculté des Sciences et Techniques, Université Cheikh Anta Dio de Dakar, B. P. 5005, Dakar Fann, Sénégal. \\ ${ }^{2}$ Laboratoire d'Agroforesterie et d'Écologie (LAFE), Département d'Agroforesterie, \\ Université Assane Seck de Ziguinchor, B.P. 523, Ziguinchor, Sénégal. \\ *Auteur correspondant, E-mail: aissatou_ne@yahoo.fr; Tél : +221 779464105 ; B.P. 523, Ziguinchor, \\ Sénégal.
}

\section{RESUME}

En Casamance, les palmeraies occupent une place primordiale dans l'équilibre écologique mais la pression anthropique, la baisse de la pluviométrie et la salinisation des sols ont affecté cet écosystème. Le présent travail a été entrepris pour étudier le comportement des jeunes plants d'Elaeis guineensis en milieu salin. Le dispositif expérimental comprend quatre parcelles élémentaires en blocs randomisés répétées quatre fois. Les plants ont été maintenus en conditions naturelles et irrigués avec une gamme de solution de $\mathrm{NaCl}(0$; $4 ; 10 ; 35$ g.l-1). Après 6 mois de traitement, les résultats ont montré une hauteur moyenne T0 $(230,44)$; T1 $(109,13)$; T2 $(90,81)$ et T3 $(78,125)$. Le taux de survie a diminué dans le temps. Il a été de 79,6\% et 50,1\% à trois mois pour T2 et T3 respectivement. Aucune mortalité n'a été observée pour T1. A 4 mois, il a été de 36 $\%$ pour $\mathrm{T} 2$ et $0 \%$ pour T3. A 5 mois, les premières mortalités ont été enregistrées pour T1 (5,3\%). Cependant, aucune différence significative n'a été observée entre T0 (100\%) et T1 $(94,7 \%)$. Le nombre moyen de feuilles par plant a été de T0 $(5,75)$; T1 $(5,5) ;$ T2 $(4)$ et T3 $(3,2)$.

(c) 2016 International Formulae Group. All rights reserved.

Mots clés : Elaeis guineensis Jacq, Basse Casamance, jeunes plants, sel, concentration, comportement.

\section{The effect of salt on the behavior of young oil palm seedlings (Elaeis guineensis Jacq.) in Lower Casamance}

\begin{abstract}
In Casamance, palm groves occupy a prominent place in the ecological balance but human pressure, declining rainfall and soil salinization affected this ecosystem. The present work aimed at studying the behavior of seedlings Elaeis guineensis in saline environment. The experimental device consists of four individual plots in randomized block repeated four times. The study was conducted during six month in natural and irrigated conditions with a range of $\mathrm{NaCl}(0 ; 4 ; 10 ; 35$ g.1-1). The results showed an average height $\mathrm{T} 0$ $(230.44 \mathrm{~cm})$; T1 $(109.13 \mathrm{~cm})$; T2 $(90.81 \mathrm{~cm})$ and T3 $(78.125 \mathrm{~cm})$. The survival rate has also decreased over time. It was 79.6 and $50.1 \%$ at three months respectively for T2 and T3 and no mortality was observed for T1. At 4 months, it was $36 \%$ for $\mathrm{T} 2$ and $0 \%$ for T3. At 5 months, the first deaths were recorded in T1 $(5.3 \%)$. However, no significant difference was observed between T0 (100\%) and T1 $(94.7 \%)$ at the 5\% threshold. The average number of leaves per plant was $5.75 ; 5.5 ; 5 ; 3.2$ respectively for T0; T1; T2 and T3.

(C) 2016 International Formulae Group. All rights reserved.
\end{abstract}

Keywords: Elaeis guineensis Jacq, Lower Casamance, seedlings, salt concentration, behavior. 


\section{INTRODUTION}

Le palmier à huile est la première plante oléagineuse au monde (Bergert, 2000). Il représente plus du tiers de la production mondiale en huiles végétales (Hoyle et Levang, 2012). Il a une productivité de près de 4 tonnes d'huile (palme + palmiste) par hectare et par an soit 7 à 10 fois plus que ses concurrents directs cultivés dans les pays tempérés (soja, colza et tournesol) (Jacquemard et al., 2011).

En Afrique et en Asie, après l'indépendance, de projets de développement rural agricole, basés sur la culture industrielle du palmier à huile, ont vu le jour (Rafflegeau, 2008). Au Sénégal, il n'existe pas de véritables palmeraies industrielles. Les peuplements de palmier à huile, estimés à 50 000 hectares, sont essentiellement naturels et localisés sur les rives du fleuve Casamance, dans le Sud du pays (FAO, 2014). Le vif intérêt porté au palmier à huile par les populations locales, pour les fruits (huiles et noix) et le vin, expliquent sa présence à proximité des villages et son extension dans les terrains de cultures. Les palmeraies naturelles sont le résultat d'un aménagement de longue date. De nombreux palmiers ont été préservés dans des zones boisées défrichées pour l'agriculture. Le palmier est l'une des premières plantes à l'état sauvages dans la région.

$\mathrm{Au}$ niveau de l'espace UEMOA, le palmier à huile est la première sous filière de la filière oléagineuse. Elle a réalisé à elle seule un chiffre d'affaire de 238 milliards FCFA en 2002, plus élevé que celui des sous filières graines de coton et arachide réunies. Elle mobilise également la moitié de la capacité de trituration industrielle (BOAD, 2008). Selon une étude menée par Hirsch (2002), les besoins en huiles végétales des pays de l'UEMOA à l'horizon 2020 devraient augmenter de plus de $70 \%$ par rapport à ceux de 1999.

Aujourd'hui, ces potentialités qu'offrent les palmeraies (ANSD, 2010) sont de plus atténuées par des contraintes d'ordre climatiques et anthropiques (PFS, 2014). L'intensification des activités humaines telles que l'irrigation, l'exploitation anarchique des ressources ainsi que des facteurs d'ordre environnementaux (remontée des nappes phréatiques salées, l'invasion marine, etc.) ont affecté la nature des sols devenus de plus en plus impropres aux activités dévouées.

La salinisation des terres est devenue une problématique mondiale (Legros, 2009, Thouraya et al., 2013). Aujourd'hui, 20\% des terres cultivées, $50 \%$ des terres irriguées sont affectées par la salinisation dans le monde (Jabnoune, 2008).

En Afrique, près de 40.000 .000 ha sont affectés par la salinisation, soit près de $2 \%$ de la surface totale (IPTRID/FAO/CISEAU, 2006).

Le Sénégal, à l'instar de tous les pays sahéliens, a subi depuis plusieurs décennies, une baisse de sa pluviométrie et des pressions de plus en plus importantes sur les ressources naturelles. Les effets combinés de la croissance démographique et les perturbations climatiques ont affecté le système bioproductif et induit la dégradation des terres. Selon LADA (2009), la dégradation des sols par salinisation affecte plus de 1,700 millions d'hectares de terre au Sénégal. En Casamance, l'avancée de la langue salée explique la salinisation et l'acidification des terres surtout au niveau des vasières et de certains bas-fonds où poussent naturellement les palmiers à huile. Cette dégradation de sols combinée à l'action anthropique a fortement affecté la végétation de la région (PFS, 2014). Des travaux ont été menés sur les comportements des espèces ligneuses (les acacias sp, Bauhinia rufesen, Eucalyptus sp) face à la salinisation (CNRF, 2011). Mais peu d'études ont été entreprises sur le comportement de l'Elaeis guinensis jacq jusqu'ici notamment en Casamance, face à la salinisation. C'est dans ce cadre que s'inscrit notre travail qui vise à étudier l'effet du sel $(\mathrm{NaCl})$ sur la croissance de jeunes plants d'Elaeis guineensis. Il cherche plus spécifiquement à déterminer le comportement des jeunes plants sous différentes concentrations de sel.

\section{MATERIEL ET METHODES \\ Site de l'étude}

L'étude a été menée dans la commune de Niaguis qui couvre une superficie de 160 $\mathrm{km}^{2}$. Elle est limitée au Nord par le fleuve Casamance, au Sud par la commune de Boutoupa Camaracounda, à l'Est par la commune d'Adéane et à l'Ouest par l'arrondissement de Nyassia (Figure 1). Le 
climat est de type soudano-guinéen caractérisé par une saison sèche qui s'étale de novembre à mi-juin et une saison des pluies allant de mijuin à octobre. La moyenne annuelle de la pluviométrie cumulée est de $1120 \mathrm{~mm}$ pour un nombre moyen de 70 jours de pluies (SEPAM, 2010). La population en 2010 a été estimée à 17120 personnes et devrait atteindre 23882 personnes en 2015 (SEPAM, 2010). La composition ethnique de la commune est très diversifiée. On y rencontre des Diolas, des Mandingues, des Mancagnes, des Manjacques, des Bainouncks, des Peuls et des Balantes. Elle compte 13 villages administratifs. Les prélèvements des plantules ont été principalement effectués dans les palmeraies des villages de Boulome et Soukouta.

\section{Matériel végétal}

Des plants ont été prélevés dans les villages de Boulome et Sououta dans la commune rurale de Niaguis Ziguinchor situé entre $12^{\circ} 33^{\prime} 40^{\prime}$ 'nord et $16^{\circ} 174^{\prime} 00^{\prime}$ 'Ouest (Figure 1). Il s'agit des endroits très peu fréquentés par le bétail, une sorte de pépinières naturelles. Les plantules saines ayant deux feuilles maximum (Photo 1) et portant des graines de palmistes ont été sélectionnées et transférées dans des gaines le même jour.

\section{Conditions d'élevage}

Les plants ont été élevés dans des sachets plastiques $(30 \quad x 15 \quad \mathrm{~cm})$ en polyéthylène que nous avons rempli avec du sable forestier prélevé dans les vergers sous les manguiers après avoir dégagé la litière. Les gaines ont été placées sous toile en plastique transparente pour éviter l'infiltration des eaux de pluies pendant le traitement. Les plants ont dans un premier temps reçu un apport d'azote (urée) à raison de $2 \mathrm{~g} / \mathrm{mois}$. L'élevage a duré 7 mois avec un apport d'eau quotidien de 0,25 litre d'eau par plant et par jour. La phase d'élevage est suivie par le traitement à l'eau salée à raison de 0,25 litre par jour et par plant lorsque les plants sont devenus rigoureux et bien développés après 7 mois d'élevage et d'entretien (Photo 2).

\section{Conduite de l'essai}

Quatre (4) gammes de concentration de l'eau salée ont été utilisées au cours du traitement: $\mathrm{T} 0$ témoin $=$ eau douce $; \mathrm{T} 1=4 \mathrm{~g} / 1$; $\mathrm{T} 2=10 \mathrm{~g} / 1$ et $\mathrm{T} 3=35 \mathrm{~g} / \mathrm{l}$. Le traitement T3 est équivalent à la concentration de l'eau de mer. Le dispositif expérimental (Figure 2) est un bloc aléatoire complet à 4 répétitions dont les parcelles élémentaires mesurent $1 \mathrm{~m}$ de long et $1 \mathrm{~m}$ de large soit une superficie utile de $1 \mathrm{~m}^{2}$ par parcelle élémentaire. Chacune des parcelles élémentaires compte 4 plants pour chaque traitement soit 16 plants pour chacune des traitements.

\section{Observations et mesures}

Les mesures ont été faites toutes les semaines. Les paramètres mesurés sont la hauteur mesurée à partir du collet jusqu'au sommet de la feuille la plus développée. Les observations ont consisté à apprécier le comportement physiologique des plants vis-àvis des différents traitements. La biomasse a été évaluée à la fin de l'essai. La matière sèche des feuilles et de racines a été calculée par la formule $\mathrm{TE}=100 \mathrm{x}(\mathrm{PF} / \mathrm{PS}) \mathrm{PF}$. La hauteur maximale, obtenue par le cumule des hauteurs au cours de l'essai pour chacun des traitements, a été appréciée à la fin de l'expérimentation.

\section{Traitements des données}

Les données sont traitées sur Excel et l'analyse statistique par le logiciel Sigma Stat (version 2.0. Jandel corporation 1992). Les comparaisons des moyennes ont été effectuées par l'analyse de variance (test d'ANOVA) suivi du test $\mathrm{t}$ de Student quand les différences étaient significatives. Les tests des coefficients de corrélation de Pearson ont été réalisés pour étudier la tendance évolutive des différents paramètres. Dans tous les cas, les différences ont été considérées significatives quand $\mathrm{p}<0,05$.

\section{RESULTATS}

Effet de la salinité sur le phénotype des jeunes plants de palmiers

Les résultats des traitements à différentes concentrations de sel ont permis d'observer que le desséchement dû à l'effet du sel est observé dans un premier temps sur les plus jeunes feuilles. Ce phénomène est observé chez tout plant traité avec de l'eau salée. Le desséchement de la plante est précédé par un rétrécissement de la surface foliaire et un changement de couleur du vert 
au grisâtre qui s'étend progressivement sur les jeunes feuilles puis sur toute la plante (Photo $3)$.

La photo 4 présente les plants T1 après 3 mois de traitement. Ces plants présentent un comportement normal comparé aux plants $\mathrm{T} 2$ et T3. Après trois mois d'irrigation à l'eau salée à la concentration $\mathrm{T} 2=10 \mathrm{~g} / \mathrm{l}$ et $\mathrm{T} 3=$ $35 \mathrm{~g} / \mathrm{l}$, les jeunes plants commencent à subir les effets du sel. Ce phénomène se traduit par un desséchement grisâtre qui s'observe tout d'abord au niveau de l'extrémité des jeunes feuilles pour s'étendre par la suite sur la plante entière.

\section{Effet de la salinité sur la croissance}

La Figure 3 montre la hauteur moyenne des plants en fonction des traitements. La hauteur des plants varie en fonction des différentes concentrations de sel. La hauteur maximale $(230,44 \mathrm{~cm})$ est observée pour le traitement $\mathrm{T} 0$ et la plus petite hauteur pour le traitement T3 $(78,125 \mathrm{~cm})$. L'analyse de la variance de la variable hauteur moyenne montre qu'il existe une différence significative entre les traitements (ANOVA, F $=14,786, \mathrm{p}<0,001)$. La comparaison des hauteurs moyennes par traitement montre que celle de $\mathrm{T} 0$ est significativement supérieure à celles observées respectivement pour T1, T2 et T3. Par contre, aucune différence significative n'est observée entre les moyennes pour $\mathrm{T} 1, \mathrm{~T} 2$ et $\mathrm{T} 3$.

Effet du sel sur la hauteur des plants selon la durée traitement

La Figure 4 représente la fonction logarithmique de la hauteur des plants en fonction de la durée du traitement. Le coefficient de la pente de la courbe T0 $(119,5$, $\left.\mathrm{R}^{\mathbf{2}}=0,935\right)$ est environ quatre fois plus importante que celle de T1 $\left(33,1, \mathrm{R}^{2}=0,969\right)$. De même, pour la courbe T3 $\left(13,5, \mathrm{R}^{2}=\right.$ $0,876)$, le coefficient de la pente représente la moitié de celle de T2 $\left(24,9, \mathrm{R}^{2}=0,899\right)$. L'analyse montre qu'il existe une différence significative entre T0 et les autres traitements statistique (ANOVA, $\mathrm{p}<$ 0,001 Par contre aucune différence significative n'est observée entre $\mathrm{T} 1, \mathrm{~T} 2$ et $\mathrm{T} 3$.

\section{Effet du sel sur l'émission foliaire}

La Figure 5 donne la production moyenne par plant en fonction de traitement.
L'analyse de la figure montre que la production maximale pour $\mathrm{T} 0$ double celle de T3 avec respectivement $(5,75)$ et $(3,2)$. Les plants pour T1 ont en moyenne $(5,5)$ et T2 (4). Cependant, aucune différence significative n'est observée entre les différents traitements (ANOVA, p = 0,084)

\section{Interaction entre la durée, la hauteur et l'émission foliaire}

La Figure 6 exprime l'interaction entre les paramètres et la durée du traitement. La croissance est régulière avec le témoin de 1 à 6 mois traitement T0 par contre, pour le traitement $\mathrm{T} 2$ et $\mathrm{T} 3$, la courbe se stabilise entre 2 et 3 mois. Le traitement T1 enregistre une croissance relativement faible par rapport au témoin. L'analyse statistique au seuil de signification $\alpha=0,05$ (ANOVA, $\mathrm{F}=6,13$; $\mathrm{p}<0,001)$ montre qu'il existe une différence significative dans l'interaction entre la durée et le traitement sur la hauteur des plants (Figure 6a). En b) La variation du taux de survie de T0 et T1 n'a pas été affectée durant les quatre (4) premiers mois période au cours de laquelle tous les plants T3 n'ont pas survécus et il n'existe que $12,5 \%$ de T2. Les résultats du test d'ANOVA montrent que ( $\mathrm{F}=$ 23,6; $\mathrm{p}<0,001)$ l'interaction entre la durée et le traitement sur le taux de survie est significative au seuil de signification $\alpha=0,05$ par contre non significative sur l'émission foliaire $(F=0,86 ; p=0,569)$.

\section{Corrélation entre les variables}

Le test des coefficients de corrélation de Pearson (Tableau 4) entre les variables Hauteur, Taux de survie et émission foliaire montre que la hauteur est corrélée positivement $(\mathrm{r}=0,588, \mathrm{p}<0,017)$ au taux de survie lui-même corrélé avec la variable émission foliaire $(r=0,675, p<0,004)$. Il n'existe pas de corrélation significative entre la hauteur et l'émission foliaire $(r=0,364$, $\mathrm{p}=0,166$ ).

\section{Effet du sel sur la mortalité}

La Figure 7 présente la variation du taux de survie des plants en fonction de la durée de traitement. Après 2 mois, les plants T3 se caractérisent par un taux de survie plus faible $(68,75 \%)$ par rapport aux plants T2 $(90,6 \%$ et T1 $(100 \%)$ (Figure $7 \mathrm{a})$. L'analyse statistique montre que la différence est 
significative entre les traitements (ANOVA, $\mathrm{p}=0,012)$. T3 a un taux de survie inférieur à celui des autres T1, T2 et T0 (Student ( $\alpha=$ 0,05). La même tendance a été observée trois mois après (Figure $7 \mathrm{~b}$ ) avec un taux de survie de 79,6 et $50,1 \%$ respectivement pour $\mathrm{T} 2$ et T3 alors que les T1 restent toujours vivants. Le taux de survie de T2 (36\%) après 4 mois (Figure 7c) le diffère significativement de T1 et T0 (ANOVA, p< 0,001). Les T1 ont commencé à enregistrer des mortalités 5 mois après l'essai (Figure 7d). L'analyse statistique (ANOVA, $\mathrm{p}<0,001)$ montre que la différence n'est pas significative entre T0 $(100 \%)$ et T1
$(95,3 \%)$. Par contre, les deux traitements diffèrent après 6 mois (Figure 7e).

\section{La tolérance à la salinité des plants de palmiers à huile}

Les plants $\mathrm{T} 1$ après 6 mois de traitement sont soumis à un régime hydrique sans sel. Ils ont repris une croissance normale caractérisée par l'élongation et l'ouverture de la plus jeune feuille (Photo 5). Sur les six plants T1 arrosés à l'eau de robinet, trois ont repris leur croissance un mois après l'arrêt du traitement au sel. Quant aux autres traitements (T2 et T3), il n'y a pas eu de reprise.

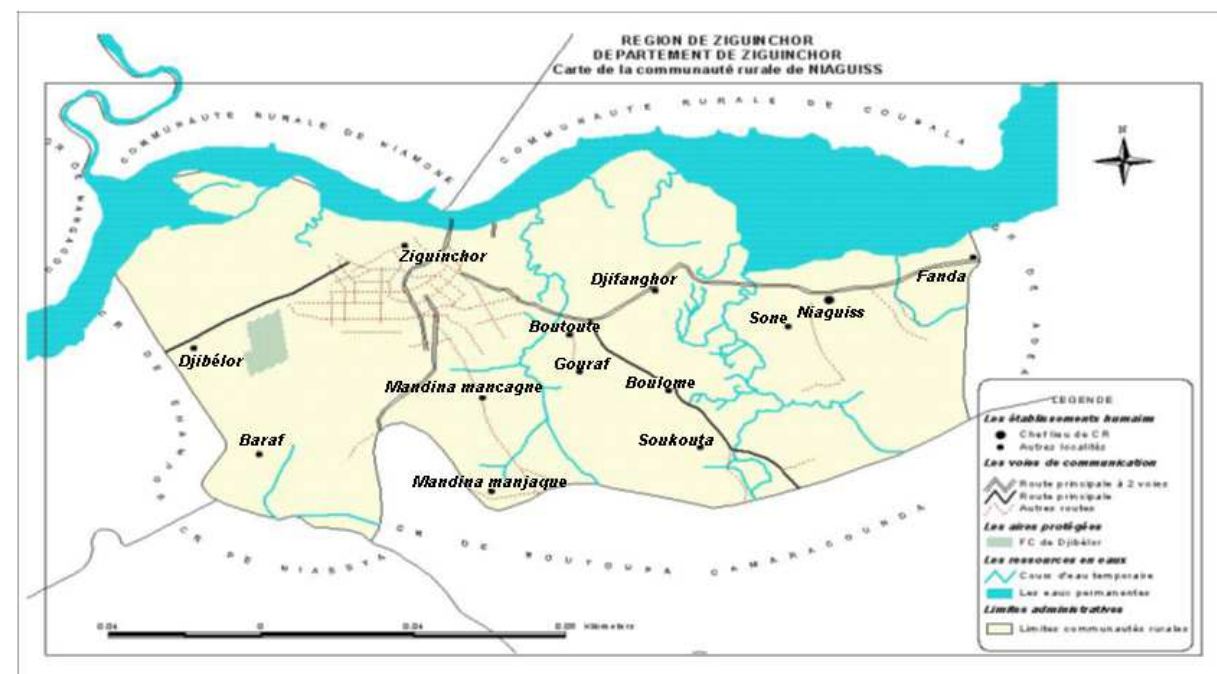

Figure 1: Carte de la commune rurale de Niaguis.

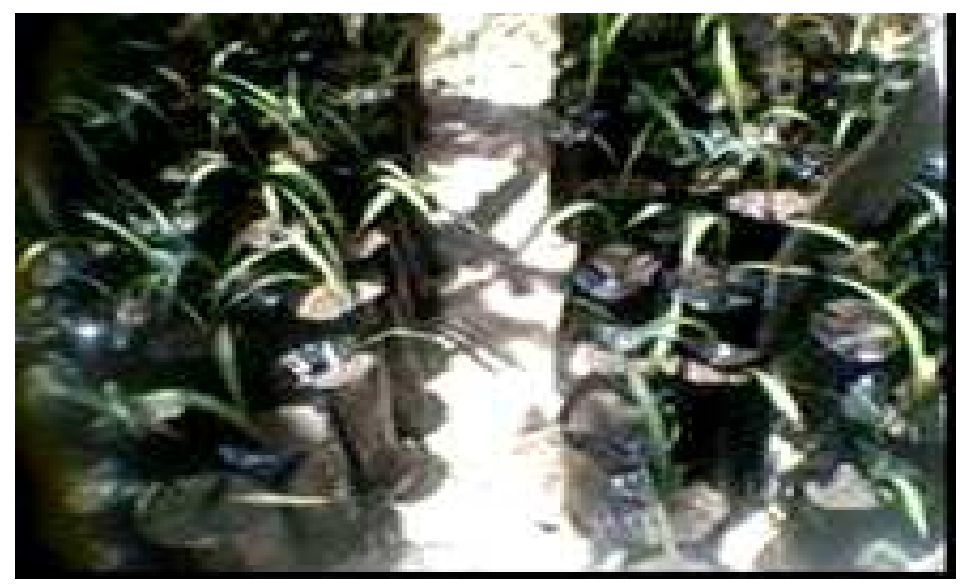

Photo 1 : Jeunes plants repiqués. 
A. D. LABO et al / Int. J. Biol. Chem. Sci. 10(3): 1312-1328, 2016

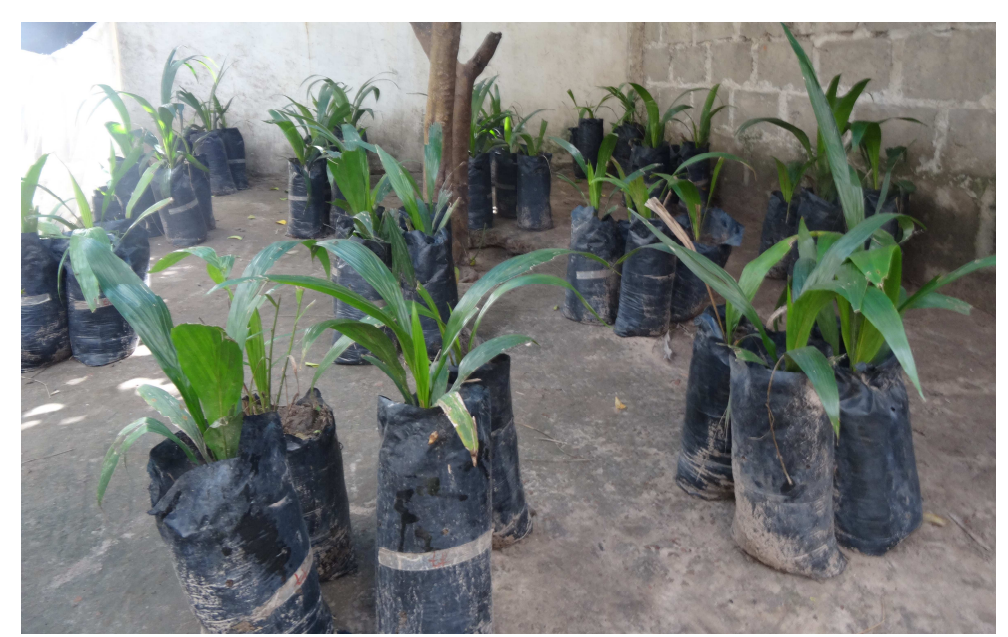

Photo 2 : Plants de palmiers après 7 mois d'élevage.

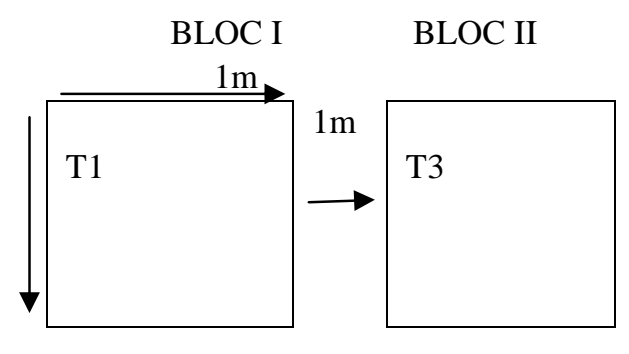

BLOC III BLOC IV
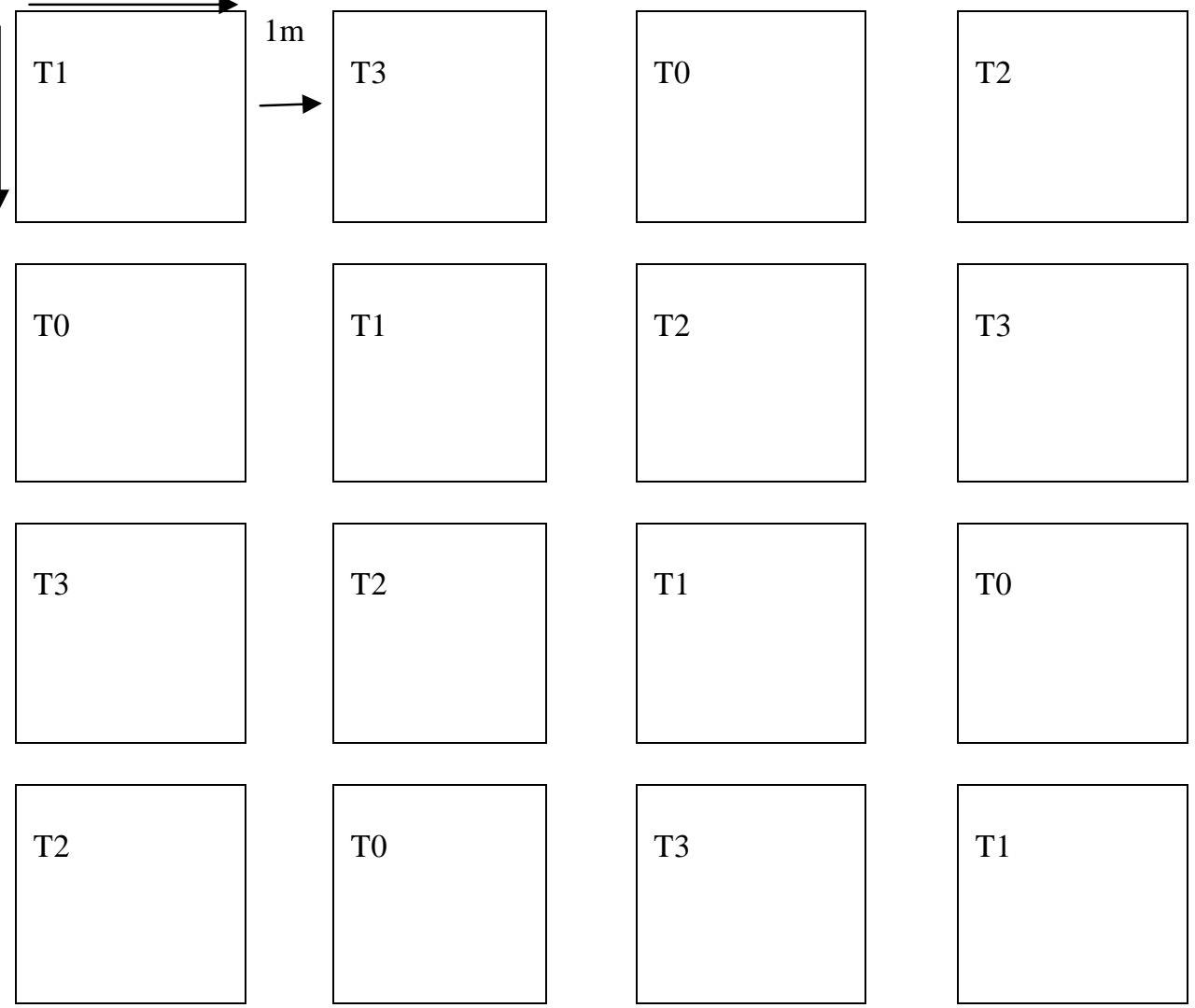

Figure 2 : Plan de l'essai. 


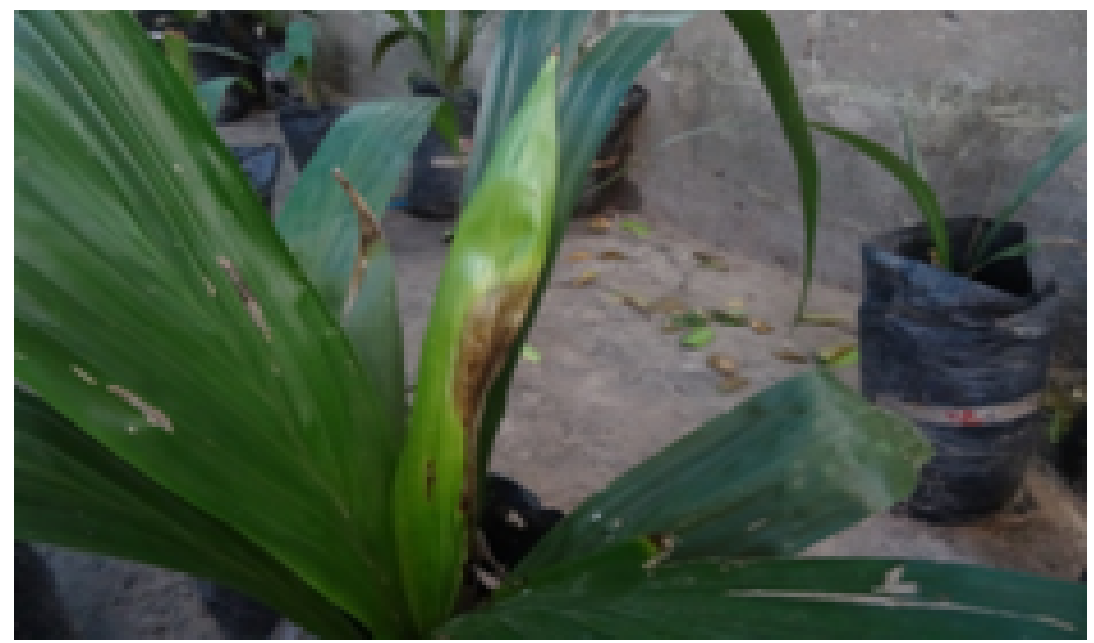

Photo 3 : Début d'apparition de nécrose brulure.

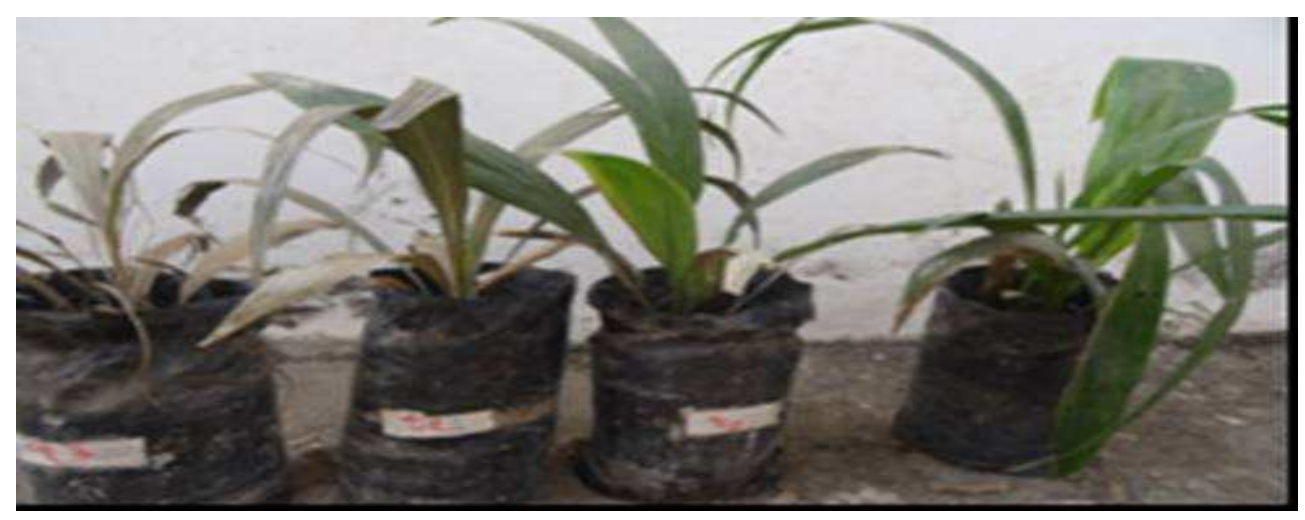

Photo 4 : Aspect des plants après trois mois de traitement.

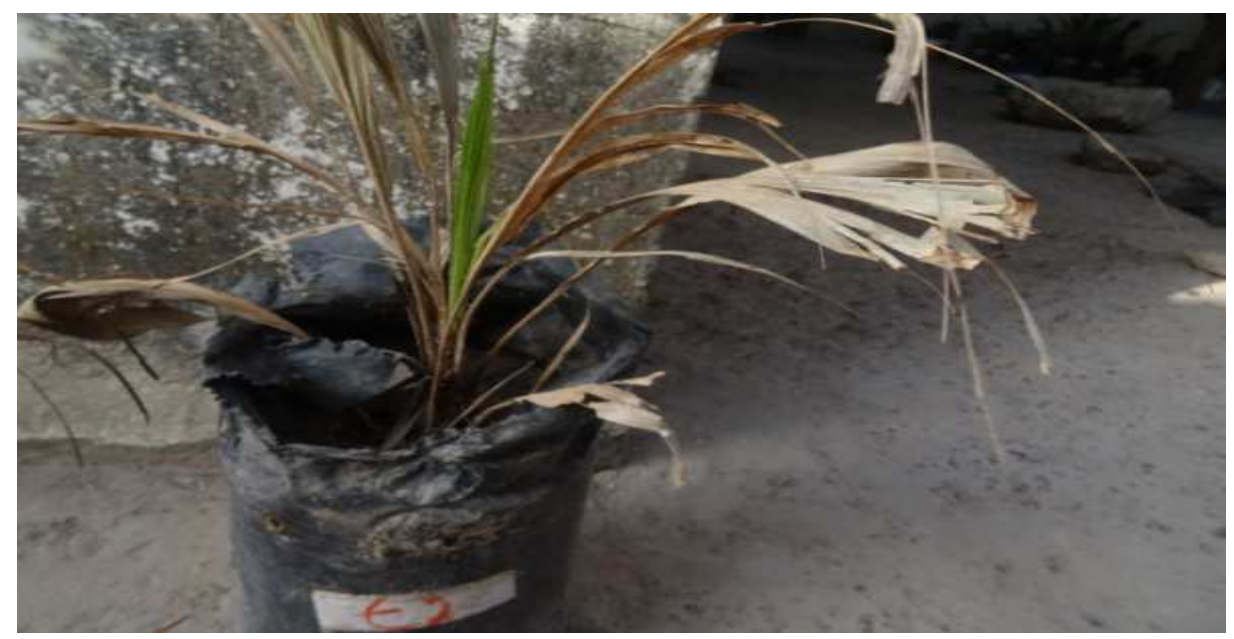

Photo 5 : Reprise de croissance de plant T1 après 6 mois de traitement. 


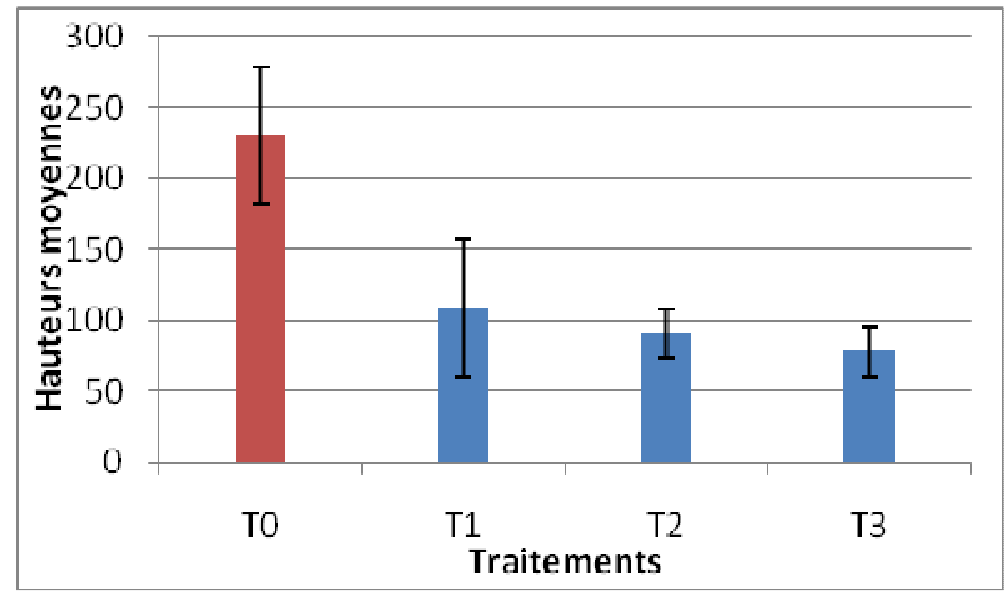

Figure 3 : Variation de la hauteur moyenne en fonction du traitement.

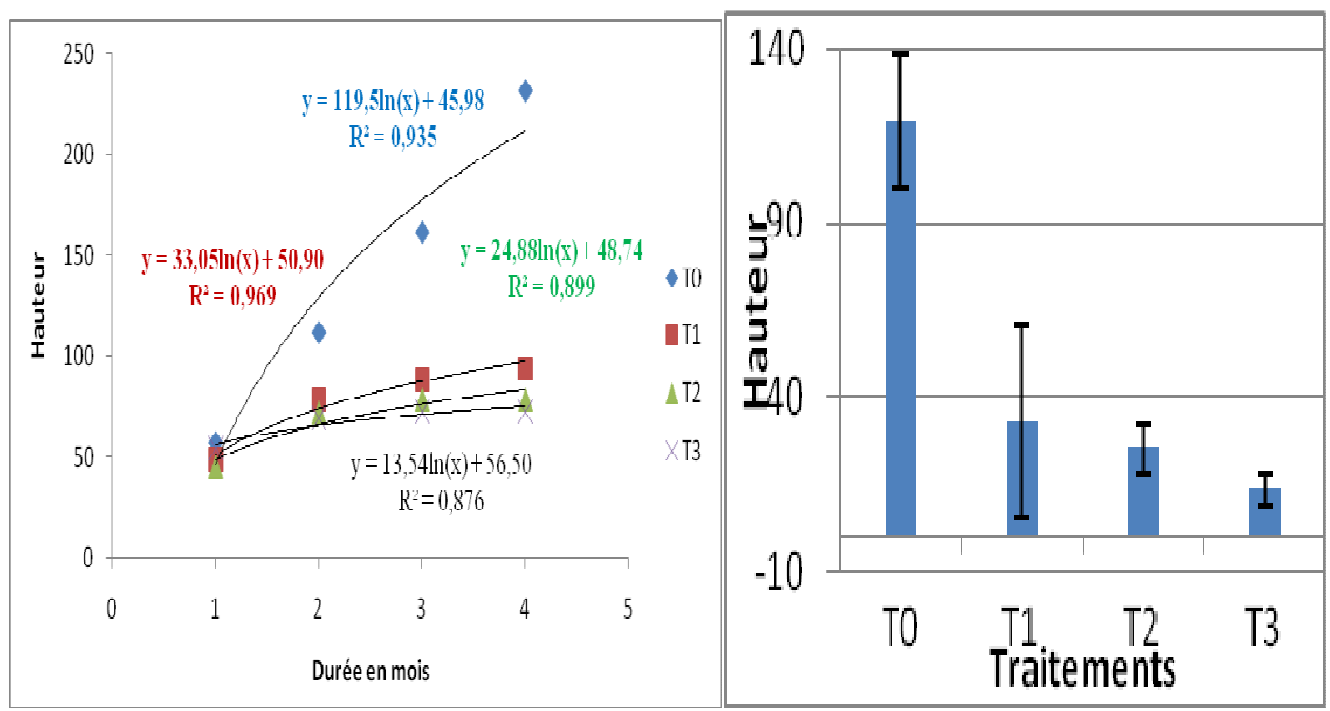

Figure 4 : La courbe logarithmique de la hauteur en fonction de la durée.

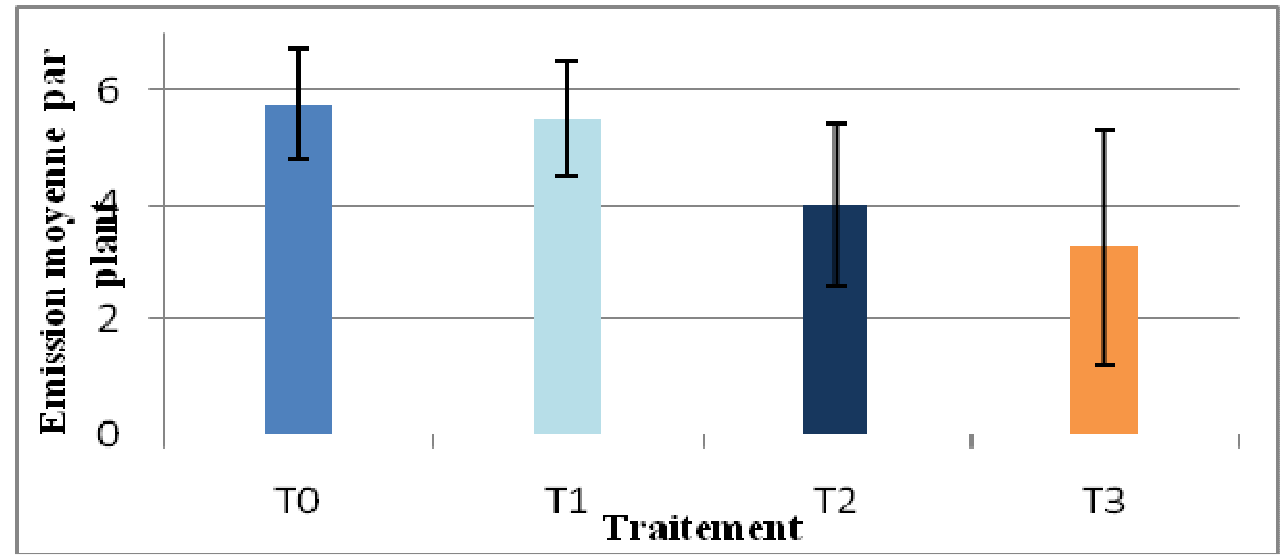

Figure 5 : Emission des feuilles en fonction de la concentration en sel. 


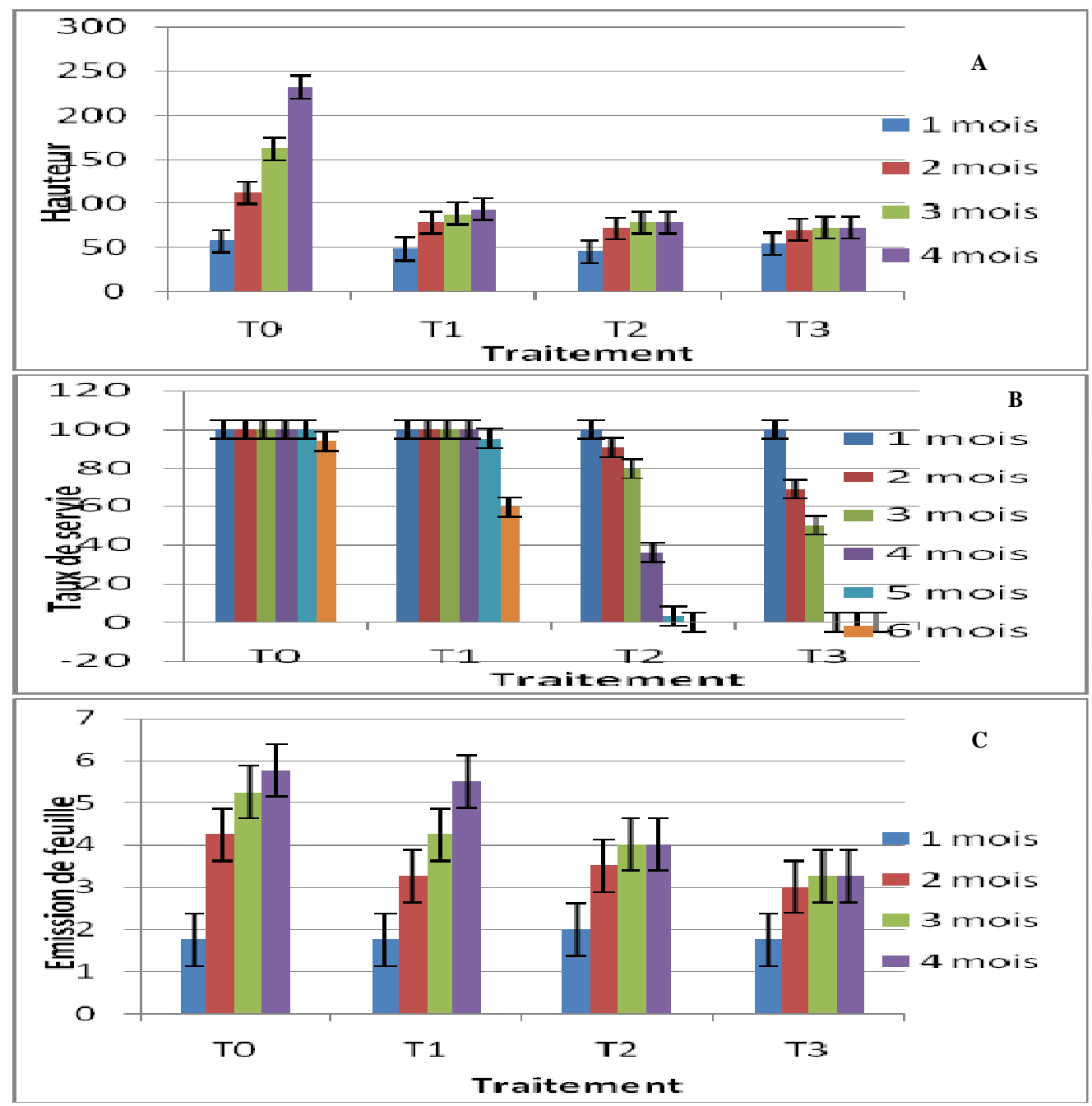

Figure 6 : Variation de la hauteur (a) du taux de survie (b) et de l'émission foliaire (c) en fonction de la durée et du traitement.

a)

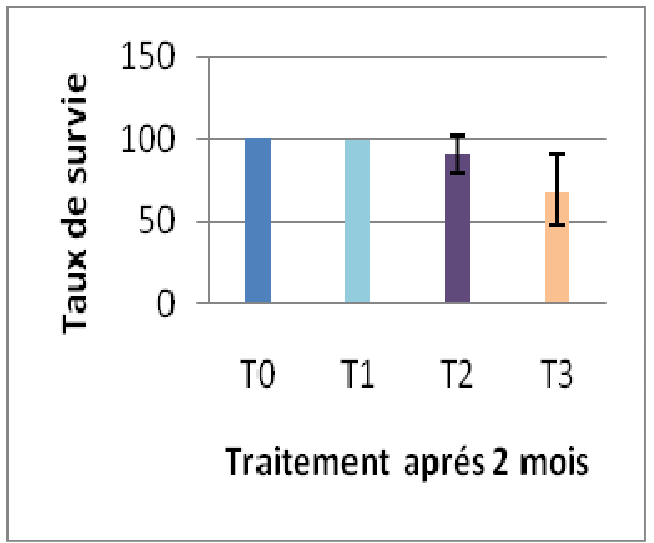

c) b)

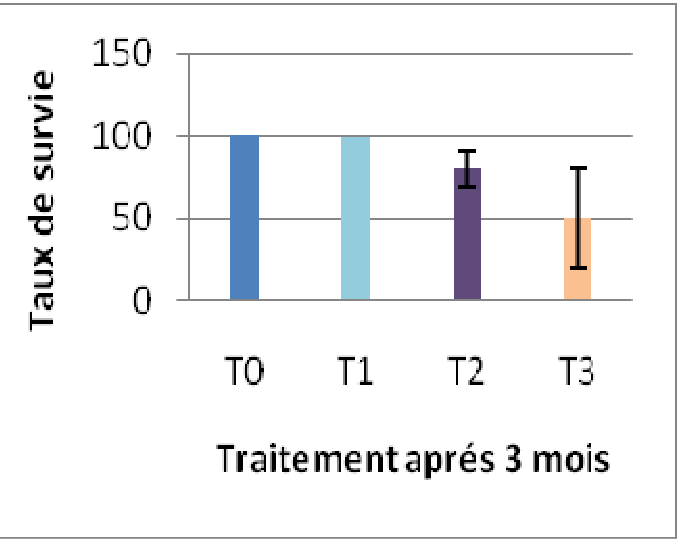

d) 

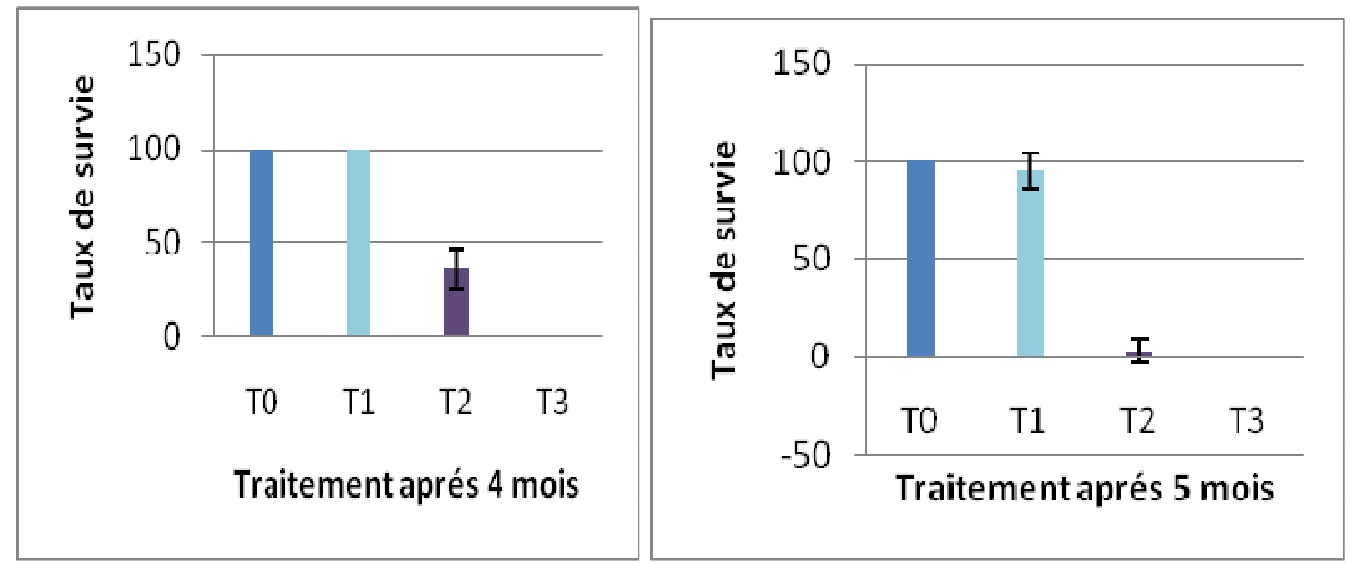

e)
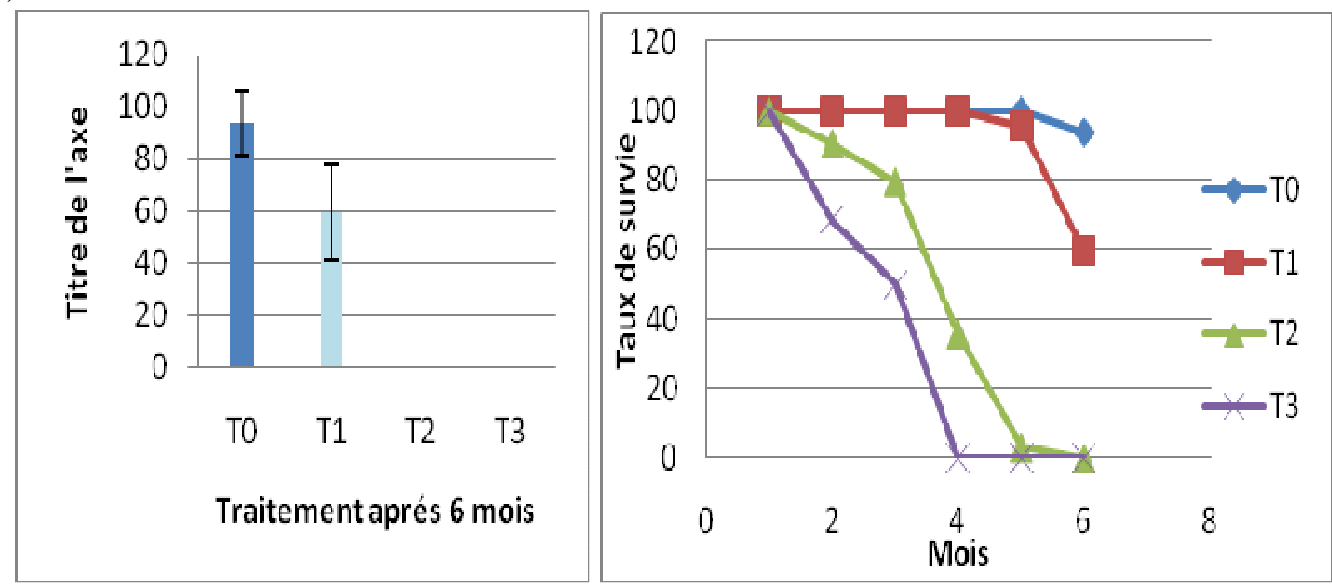

Figure 7 : Variation du taux de survie des plants soumis à des doses croissantes de $\mathrm{NaCl}$ en fonction de la durée de traitement.

Tableau 1 : Analyse de la variance pour la variable hauteur moyenne avec comparaison par Test de Student Newman Keuls.

\begin{tabular}{llccccc}
\hline Source de Variation & DF & SS & MS & F & P & \\
Entre Groupes & 3 & 58868,3 & 19622,76 & 14,786 & $<0,001$ & \\
Résiduelle & 12 & 15925,5 & 1327,122 & & & \\
Total & 15 & 74793,8 & & & & \\
\hline Comparaison & Différence de Moyenne & $\mathbf{p}$ & $\mathbf{q}$ & $\mathbf{P}$ & $\mathbf{P}<\mathbf{0 , 0 5 0}$ \\
T0 vs. T3 & & 152,313 & 4 & 8,362 & $<0,001$ & S \\
T0 vs. T2 & 139,625 & 3 & 7,665 & $<0,001$ & S \\
T0 vs. T1 & 121,313 & 2 & 6,66 & $<0,001$ & S \\
T1 vs. T3 & 31 & 3 & 1,702 & 0,474 & NS \\
T1 vs. T & & 18,313 & 2 & 1,005 & 0,49 & NS \\
T2 vs. T & 12,688 & 2 & 0,697 & 0,63 & NS \\
\hline
\end{tabular}


A. D. LABO et al / Int. J. Biol. Chem. Sci. 10(3): 1312-1328, 2016

Tableau 2 : Analyse de la variance pour la variable taux de survie après 6 mois.

\begin{tabular}{cccccc}
\hline Entre Groupes & 3 & 25810,547 & 8603,516 & 67,769 & $<0,001$ \\
Résiduelle & 12 & 1523,438 & 126,953 & & \\
Total & 15 & 27333,984 & & & \\
\hline Comparaison & Différence de Moyennes & $\mathrm{p}$ & $\mathrm{q}$ & $\mathrm{P}$ & $\mathrm{P}<0,050$ \\
T0 vs. T3 & 93,75 & 4 & 16,641 & $<0,001$ & $\mathrm{~S}$ \\
T0 vs. T2 & 93,75 & 3 & 16,641 & $<0,001$ & $\mathrm{~S}$ \\
T0 vs. T1 & 34,375 & 2 & 6,102 & 0,001 & $\mathrm{~S}$ \\
T1 vs. T3 & 59,375 & 3 & 10,539 & $<0,001$ & $\mathrm{~S}$ \\
T1 vs. T2 & 59,375 & 2 & 10,539 & $<0,001$ & $\mathrm{~S}$ \\
T2 vs. T3 & 0 & 2 & 0 & 1 & $\mathrm{NS}$ \\
\hline
\end{tabular}

Tableau 3: ANOVA 2 sur la Hauteur, le taux de survie et l'émission Foliaire.

\begin{tabular}{lccccccccccccccc}
\hline Source of Variation & \multicolumn{1}{c}{ SC } & \multicolumn{1}{c}{ DDL } & & CM & F & P \\
\hline Durée & Ha & TS & EF & Ha & TS & EF & Ha & TS & EF & Ha & TS & EF & Ha & TS & EF \\
Traitement & 39783 & 47858 & 73,31 & 3 & 5 & 3 & 13261,1 & 9571,7 & 24,4 & 20,8 & 98,3 & 16 & $<0,001$ & $<0,001$ & $<0,001$ \\
Durée x Traitement & 59655 & 67370 & 17,31 & 3 & 3 & 3 & 19884,9 & 22457 & 5,77 & 31,2 & 231 & 3,77 & $<0,001$ & $<0,001$ & 0,017 \\
& 35207 & 34239 & 11,81 & 9 & 15 & 9 & 3911,94 & 2282,6 & 1,31 & 6,13 & 23 & 0,86 & $<0,001$ & $<0,001$ & 0,569 \\
\hline
\end{tabular}

NB : Ha ( hauteur), TS (taux de survie), EF (émission foliaire), DDL (degré de liberté), SC (somme des carrés), CM (variance ), $\mathrm{F}$ (valeur limite), $\mathrm{P}$ ( seuil de probabilité) S (significatif ), NS (non significatif).

Tableau 4 : Corrélation de Pearson entre les variables.

\begin{tabular}{lcc}
\hline & Taux Survie & Emission foliaire \\
\hline Hauteur & 0,588 & 0,364 \\
& 0,0165 & 0,166 \\
& 16 & 16 \\
Taux de Survie & & 0,675 \\
& & 0,00409 \\
& 16 \\
Emission foliaire & & \\
\hline
\end{tabular}




\section{DISCUSSION}

\section{Symptomatologie}

Pour apporter les données scientifiques nécessaires à l'interprétation des actions réciproques entre la plante et son milieu, il faut connaître au préalable le déroulement des phénomènes morphologiques qui expriment les modifications physiologiques de l'organisme (Hanana et al., 2011) . Chez Elaeis guineensis, les ions $\mathrm{Na}+$ provenant de solution du sel et absorbés par la plante s'accumulent préférentiellement dans les jeunes feuilles. Le principe de mobilisation des éléments nutritifs chez le jeune palmier est particulier (des racines aux feuilles car les jeunes palmiers ne possèdent pas encore de tige). L'analyse foliaire est apparue très tôt comme un élément clé du suivi de l'état nutritionnel des palmiers. Les teneurs optimales varient en fonction de l'âge, du matériel végétal, du climat, de certaines techniques culturales, de la densité et des équilibres entre éléments minéraux. Toute valeur optimale ne peut donc être représentative d'une situation déterminée (Thomas et Jean, 2001). Il semble qu'une augmentation de teneur en $\mathrm{K}+$ sl'accompagne d'un relèvement de la teneur en $\mathrm{Cl}$-. Les différences de feuilles sèches TS ou de teneur en potassium dans les feuilles ont varié selon le génotype, la dose de potassium et le régime hydrique.

(Nodichao et al., 2007). Comme toutes les monocotylédones, le palmier à huile possèdent des racines fasciculées capables d'explorer une grande surface pour des éléments nutritifs (Thomas et Jean, 2001). Ce système racinaire reste à quelques centimètres du sol (Jacquemard et al., 2011). Les racines comme les feuilles et les tiges se modifieront pour s'adapter à l'environnement dans lequel elles vivent. L'architecture du système racinaire du palmier à huile joue un rôle important dans la résistance à la sécheresse, grâce à la mobilisation de l'eau et des sels minéraux (Nodichao et al., 2008). La tige surtout quand elle est vivace, accumule des substances nutritives pour assurer le développement de la plante (Jacquemard et al., 2011). Le stress salin entraîne le stress hydrique. (Hanana et al., 2011; Farnoosh et Saeed, 2013). L'alimentation hydrique est le principal facteur limitant la productivité du palmier à huile (Elaeis guineensis Jacq.) (Ndiaye et al., 2007). Si la plante ne réussit pas à compenser par absorption les pertes d'eau qu'elle subit par la transpiration, le bout des tiges et les feuilles fanent. Quand cette condition persiste, les feuilles se dessèchent et meurent. Le traitement témoin n'a présenté aucun signe de toxicité par contre, les plants traités présentent des signes visibles sur le feuillage après 40 jours. Il a observé une diminution par rapport au control. (Gonzalez et al., 2012).

\section{Croissance}

La hauteur maximale de plants T3 (35 g.1-1) est le tiers de celle de Témoin T0 ellemême deux fois celle de T1 (4 g.l-1) et T2 (10 g.1-1). Le sel $(\mathrm{NaCl})$ affecte la croissance en hauteur des jeunes plants d'Elaeis guinensis Jacq. Ces résultats sont confirmés par la courbe logarithmique de la croissance en hauteur suivant la durée du traitement. La courbe exprime un rythme de croissance progressive plus accélérée pour des concentrations croissantes (T0>T1>T2>T3). Déjà à T1 (4 g.1-1), la différence est hautement significative. Le taux de réduction de la croissance en hauteur varie de $52 \%$ (pour 4 g.1-1) à $66 \%$ (pour 35 g.1-1). Les jeunes plants réagissent en adoptant un comportement de réduction de la croissance en hauteur. La croissance d'une plante dépend de plusieurs facteurs entre autre la disponibilité de l'eau et des éléments nutritifs. Ces éléments sont absorbés par les racines. Etant donné la lignification rapide des racines chez le palmier à huile, il semble que l'absorption s'effectue principalement par les jeunes radicelles, et surtout les racines de $4^{\text {ème }}$ ordre qui ne se lignifient pas (Jacquemard, 2011). Sous l'effet du sel, le pouvoir d'absorption de ces racines s'amenuise, entraînant ainsi une réduction de la croissance des jeunes plants. La salinité des sols constitue l'un des principaux stress abiotique limitant la croissance des plantes cultivées (Munns et Testy, 2008). L'émission foliaire varie légèrement suivant la concentration du sel. On a observé chez les plants T1 $(5,5)$ et T3 $(3,2)$ par rapport au témoin T0 $(5,75)$. Statistiquement, il n'y a pas de différence 
significative entre les traitements autrement dit, le sel n'affecte pas la production foliaire chez les jeunes plants d'Elaeis guinensis. Ceci est en rapport avec la durée du traitement au delà de trois (3) mois. L'émission foliaire s'effectue suivant un rythme (mensuel) et un ordre bien définie. La tolérance au sel durant plusieurs mois de traitement permet aux jeunes palmiers de reconstituer l'appareil aérien. Ce résultat confirme la corrélation positive entre le taux de survie et l'émission foliaire. La digestion de l'albumen par le suçoir n'est terminée que 4 mois après la germination. Ceci montre l'importante contribution des réserves de l'albumen dans la croissance de la jeune plantule. En stade jeune âge, ce sont les feuilles qui participent à la croissance en diamètre du stipe. La croissance longitudinale est réduite. Le stipe de palmier constitue vraisemblablement un organe de réserve important pour la nutrition hydrocarbonée et peut-être aussi pour la nutrition minérale. (Samira et al., 2011) ont montré que les taux de réduction chez Capsicum annuum L. par rapport au témoin sont de 10,$2 ; 24,6$ et $30,9 \%$ aux doses de $\mathrm{NaCl}$ 2,4 et 6 g.l-1 respectivement. Le stress salin ((o, 25, 50, $100 \mathrm{mmol}$ de $\mathrm{NaCl})$ a entraîné une augmentation significative de la teneur en $\mathrm{Na}^{+}$ des trois variétés de piment (Thouraya et al., 2013). La réduction de la hauteur des plantes en condition de salinité pourrait s'expliquer par l'inhibition de l'élongation de l'axe principal ou la diminution de la longueur des ramifications. Les plants de Jatropha curcas L. répondent aux différentes concentrations de $\mathrm{NaCl}$ par une réduction de la croissance en hauteur pour les concentrations supérieures ou égales à 4 g.l-1. Hishida et al. (2014) ont observé que les deux espèces de Jatropha curcas peuvent croître sur des salinités supérieures à $100 \mathrm{mM} \mathrm{NaCl}$ ). La réduction est significative 60 jours après. Ayoub et al. (2014) ont observé une réduction de 20 et $30 \%$ par rapport au témoin et que les jeunes plants peuvent supporter jusqu'à $160 \mathrm{mM}$ $\mathrm{NaCl}$. Il conclue que jatropha est une plante tolérante à la salinité. Chaum et al. (2013) ont étudié le stress hydrique sur la croissance de jeunes plants de Elaeis guineensis jacq. Ils ont observé une réduction significative de 11,5; 20,$9 ; 28,4$ et $41,9 \%$ pour des teneurs en eau du sol respectives de $42 ; 20 ; 13$ et $6 \%$. L'effet de la salinité sur le nombre de feuilles par plant montre que les deux provenances sont négativement affectées lorsque le traitement salin atteint les concentrations supérieures ou Comparé aux autres plantes agronomique et horticultural. La salinité réduit la croissance de citrus et cause de désordres physiologiques (Seera, 2000; Garcia et al., 2006 ; Rashad, 2011). Des effets significatifs sur la croissance et le rendement sont observés sur Verna leon (37\%), Cleopatra mandarin (46\%) et macrophylla $(56 \%)$. Siolados montre une croissance rapide chez le témoin et les plants atteignent la longueur et le poids maximum en seulement 20 jours comparé au Aeluropus lagopoides (45 jours) et 60 jours pour Urochondra setulosa (Gulzar et al., 2003a, 2003b). L'optimum de croissance de la plupart des monocotylédones halophytes est observé à $300 \mathrm{mM} \mathrm{NaCl}$. Aeluropus, Paspalum, Puccinellia, Spartina et Sporobolus de zones intertidales survivent à $540 \mathrm{mM} \mathrm{NaCl}$ pendant que les autres espèces de l'habitat ne tolèrent guère des concentrations de $\mathrm{NaCl}$ égales à 16 g.1-1 (Ly et al., 2014). Konan et al. (2015) ont mis en évidence une influence significative de la qualité morphologique des plants enracinés. La reprise dépend fortement du développement foliaire, notamment la taille des plants racinés. La bonne qualité des racines produites in vitro concourt aussi au succès de la reprise en l'acclimatation.

Les résultats sur la vitesse de croissance en hauteur de 294 arbres appartenant à 11 descendances de palmier à huile ont montré que l'ensemble des descendances testées ont une vitesse de croissance en hauteur moyenne inférieure au témoin. En effet, les valeurs obtenues ont varié de 19,86 à 35,89 cm/an au sein de ces descendances testées, contre $45,25 \mathrm{~cm} /$ an pour le témoin de l'essai. Une réduction de plus de $20 \%$ de la vitesse de croissance en hauteur a donc été observée au niveau de ces descendances (Konan et al., 2014).

\section{Taux de survie}

En ce qui concerne le taux de survie, on a enregistré un taux de survie allant jusqu'à 90 jours pour des concentrations de 35 g.l-1 et 
180 jours pour 4 g.l-1. Le taux de survie de T2 (10 g.l-1 soit $36 \%)$ après 4 mois le diffère significativement des traitements $\mathrm{T} 1$ et $\mathrm{T} 0$. Les T1 ont commencé à enregistrer des mortalités 5 mois après l'essai. L'analyse statistique montre que la différence n'est pas significative entre T0 (100\%) et T1 $(95,3 \%)$. Par contre, les deux traitements diffèrent après 6 mois. La concentration de $4 \mathrm{~g} 1$ est insuffisante pour provoquer la mortalité en quelques jours. Il faudrait des concentrations plus élevées. Deux provenances de Jatropha curcas L. supportent des concentrations de $\mathrm{NaCl}$ allant jusqu'à 35 g.l-1 pendant une durée de 42 jours (LY et al., 2014). Le taux de mortalité d'Atriplex. lentiformis (23,36 g.1-1 soit 5,56\%) après 2,5 mois (Faye et al., 2014). La salinité peut être due à plusieurs raisons mais les effets les plus marquants sont attribués à l'augmentation des ions $\mathrm{cl}$ et $\mathrm{Na}+$ (Zekri, 2001; 2004) dans les différents organes de la plante. Ces ions engendre des situations critiques pour la survie de la plante en interceptant différents mécanismes (Raveh, 2005 ; Grieve et al., 2007). Atriplex, lentiformis et $A$. nummularia produisent bien à des salinités élevées et $A$. lentiformis présente un bon potentiel de croissance avec un taux de survie de 83 à $88 \%$ comparé au taux de survie de A. nummularia (26-34\%) et A. halimus (15-23\%). Sporobolus ioclados à peine survie à $500 \mathrm{mM} \mathrm{NaCl}$ et donc paraît être une plante modérément tolérante en comparaison avec d'autres plants.

\section{La production de biomasse}

Le stress salin a provoqué une réduction de matière sèche racinaire de 16,6 ; $54 ; 55,4 \%$ respectivement pour $\mathrm{T} 1, \mathrm{~T} 2, \mathrm{~T} 3$. Quant à la matière sèche des feuilles, on a enregistré plus de $50 \%$ de réduction. La biomasse sèche de toutes les parties de la plante (feuilles, tiges et racines) des deux provenances de Jatropha curcas a été significativement plus faible à partir des concentrations de 16 g.l-1 (LY et al, 2014). La production moyenne de biomasse sèche aérienne par plante de variétés de piments est passée de 11,6 g pour le traitement témoin à $6,7 \mathrm{~g}$ en présence de 6 g.l-1 $\mathrm{NaCl}$, induisant ainsi une diminution significative de l'ordre de $42,2 \%$ (Samira et al., 2011). Le stress salin $(100,200,300,400$, et $500 \mathrm{mM} \mathrm{NaCl})$ inhibe de manière significative les poids secs, les poids frais, la longueur des feuilles et des racines et le nombre de feuilles de Sporobolus ioclados (Gulzar et al., 2005). A $120 \mathrm{mM}$ $\mathrm{NaCl}, 4$ maïs hybrides enregistrent plus de $50 \%$ de réduction de poids sec (Muhammad et al., 2010). A. lentiformis produit la biomasse fraîche de 10,3 to 26,8 t/ha comparativement à $A$. nummularia $(8,3$ à 20,4 t/ha) et $A$. halimus (2,9 à 12,6 t/ha) (Shaoib, 2013). Mandarinier Cléopâtre (Citrus reshni hort ex Tan), mandarinier commun (Citrus delicosa Ten.), pomelo Star Ruby ruby (Citrus paradisi Macfad.cv Star Ruby) et orange Shamouti (Citrus sinensis) maintenus sur des milieux MT renfermant des doses de $\mathrm{NaCl} 0$, $50,100,150$ et $200 \mathrm{mM}$ ont montré que les différents niveaux de stress de salinité ont eu un effet significatif sur la croissance des cellules (Chetto et al., 2015)

\section{Conclusion}

L'étude du comportement des jeunes plants de Elaeis guineensis jacq sous stress salin montre que les paramètres de croissance (hauteur, émission foliaire, biomasse sèche des feuilles et des racines) décroissent quand la concentration du sel augmente, indicateur de la sensibilité des jeunes plants au stress salin.

La mortalité des plants T3 (concentration sensiblement égale à celle de l'eau de mer) après trois mois est un autre indicateur de la sensibilité des jeunes plants. Ces résultats étant préliminaires, il serait opportun d'envisager d'autres recherches sur l'effet de sel sur les jeunes plants de l'Elaeis guineensis afin de confirmer les résultats.

Des études comparatives de jeunes plants de variétés Tenera, Dura, et Pisifera en conditions salines peuvent être conduites en vue d'un programme d'amélioration génétique de la résistance de palmier à huile au stress salin.

\section{CONFLIT D'INTERET}

Les auteurs déclarent qu'ils n'ont aucun conflit d'intérêts. 


\section{CONTRIBUTION DES AUTEURS}

ADL a été l'investigateur principal ; D NG a contribué à la rédaction du manuscrit ; SS a contribué à la rédaction du manuscrit; LEA a contribué à la rédaction du manuscrit.

\section{REMERCIEMENTS}

Ce travail est une initiative propre à partir du constat sur l'état des palmeraies en Casamance. Les auteurs remercient l'assistance technique du Centre National de Formation Technique des Agents des Eaux et Forets et des parcs (CNFTAEFP), Institut Sénégalais de Recherches Agricoles de Ziguinchor (ISRA), le Département de biologie végétale de l'UCAD.

\section{REFERENCES}

ANSD. 2010. Situation économique et sociale de la région de Ziguinchor, Rapport final, p. 106.

Ayoub ZE, Mohamed OG. 2014. Effect of Sodium Chloride on Growth of Jatropha (Jatropha curcas L.) young transplants. Universal Journal of Plant Science, 2(1): 19-22. DOI: 10.13189/ujps.2014.020103.

Bergert DL. 2000. Management strategies of Elaeis guineensis (oil palm) in response to located markets in south eastern Ghana, West Africa, Master of Science in forestry, Michigan Technolgical University, Ghana, p.120.

BOAD. 2008. Etude sur la promotion et le développement de la filière oléagineuse dans l'espace UEMOA sous filière palmier à huile, Rapport final, p. 271.

Chaum S, Yamada N, Takabe T, Kirdmanee C. 2013. Physiological features and growth characters of oil palm (Elaeis guineensis Jacq.) in response to reduced water-deficit and rewatering. AJCS, 7(3):432-439.

Chetto O, Dambier D, Fadli A, Bakirane R, Talha A, Beniyahia H .2015. Mise au point d'un test in vitro de comportement au sel de quatre génotypes d'agrumes. Journal of Applied Biosciences, 88. DOI : http://dx.doi.org/10.4314/jab.v88i1.1 ; http://www.ajol.info/index.php/jab/arti cle/view/117630

CNRF. 2011. La salinisation au Sénégal: une menace pour la sécurité alimentaire.http://www.cncr.org/spip.p hp?article 403.

Fairhurst T et Caliman J-P. 2001. Symptômes des déficiences minérales et anomalies chez le palmier à huile (Elaeis guineensis Jacq.). Série palmier à huile. Institut des Phosphates et de la Potasse (PPI), Singapour, et CIRAD, France, $64 \mathrm{p}$.

FAO. 2014. Etude sur les ressources forestières et les plantations forestières du Sénégal. Annuaire FAO des produits forestiers 2014, 70p.

Farnoosh Rajabi, saeed Vazan. 2013. Effect of salinity on $\mathrm{Na}+$ and $\mathrm{K}+$ compartment in salt tolerant and sensitive wheat genotypes. Scholarly Journal of Agricultural Science, 4(1): 14-23 www.scholarly-journal.com/SJAS.

Faye E, Mouhameth C, Mamoudou AT. Abdou M. 2014. Evaluation et amélioration du comportement de Atriplex lentiformis (Torr.) S. Watson en milieux salés au Sénégal. Int. J. Biol. Chem. Sci., 8(4): 1697-1709. http://dx.doi.org/10.4314/ijbcs.v8i4.30

Garcia Sanchez F, Syvertsen1 J P, Martınez V. Melgar J C. 2006. Salinity tolerance of Valencia' orange trees on rootstocks with contrasting salt tolerance is not improved by moderate shade. Journal of Experimental Botany, 57(14): 36973706. doi:10.1093/jxb/erl121

Grieve AM, Prior DL, Bevington BK. 2007. Long- term effect of saline irrigation water on growth, yield, and fruit quality of Valencia orange trees. Aus. J. Agri. Res., 58: 342-348.

Gonzalez P, James PS, Etxeberria E.2012. Sodium Distribution in Salt-stressed Citrus Rootstock Seedlings. Hortscience, 47(10): 1504-1511.

Gulzar S, Khan MA, Ungar IA. 2003a. Effects of Salinity on growth, ionic content and plant- water relations of Aeluropus lagopoides. Comm. Soil Sci. Plant Anal., 34: 1657-1668. 
Gulzar S, Khan MA, Ungar IA. 2003b. Salt tolerance of a coastal salt marsh grass. Comm. Soil Sci. Plant Anal., 34: 25952605.

Gulzar S, Ajmal KM, Irwin AU, Xiaojing Liu, 2005. Influence of salinity on growth and Osmotic relation of Sporobulus ioclados. Pak. J. Bot., 37(1): 119-129.

Hoyle D, Levang P. 2012. Le développement du palmier à huile au Cameroun WWF, IRD/CIFOR; p.16.

Hanana M, Hamrouni L, Cagnac O, Blulwald E. 2011. Mécanismes et stratégies cellulaires de tolérance à la salinité $(\mathrm{NaCl})$ chez les plantes. www.nrcresearchpress.com/er.

Hishida S. Ascencio-Valle F, Fujiyama H., Orduño-Cruz A, Endo T, Larrinaga JÁ.-Mayoral. 2013. Differential responses of jatropha species on growth and physiological parameters to salinity stress at seedlings plant stage, Communications in soil science and plant analysis. Tandfonline. com/doi/pdf, on line, august 2013.

Hirsch R. 2002. Développement l'UEMOA et les perspectives de création d'un marché régional des corps gras en Afrique de l'Ouest. Agence Française de Développement. Oléagineux, Corps Gras, Lipides, 9(4) : 199-205.

IPTRID/FAO/CISEAU. 2006. Extension de la salinisation, stratégies de prévention et rehabilitation. Conférence électronique sur la salinisation, p.12.

Jacquemard JC, André B, Jean O, Laurence O, Aude V, Jean. G, Hubert F. 2011. Le Palmier à Huile. Editions Quae, CTA, Presses agronomiques de Gembloux, 948.

Konan JN, Allou D, Diabate S, Konan EP, Koutou A. 2014. Evaluation de l'introgression du caractère croissance lente de quelques géniteurs Akpadanou (origine Bénin) chez quelques géniteurs améliorés de palmier à huile (E. guineensis Jacq.) de l'origine La Mé (Côte d'Ivoire). Int. J. Biol. Chem. Sci., 8(5): 2015-2022. DOI : http://dx.doi.org/10.4314/ijbcs.v8i5.7

Konan EK, Allou D, Diabaté S, Kone B, Hala $\mathrm{N}$, Issali AE, Yatty JK, Durand-
Gasselin T. 2015. Facteurs affectant la reprise au sevrage des vitroplants enracinés de palmier à huile (Elaeis guineensis Jacq.) régénérés par embryogènese somatique. Agronomie Africaine, 27: 1-14. http://www.ajol.info/index.php/aga/arti cle/view/118752

LADA. 2009. Evaluation de la dégradation des terres au senegal projet Fao land degradation assessment (lada). Rapport zone d'étude de Diabal-Niakha (G.A.A de Barkedji, p.56

Ly MO, Dinesh K, Mayecor D, Subhash N, Tahir D. 2014. Effet de la salinité sur la croissance et la production de biomasse de deux provenances de Jatropha curcas L. cultivés en serre. Int. J. Biol. Chem. Sci., 8(1): 46-56. DOI : http://dx.doi.org/10.4314/ijbcs.v8i1.5

Legros J.P. 2009. La salinisation dans le monde. Académie des Sciences et Lettres de Montpellier, Conférence $n^{\circ} 4069$, Bull. $n^{\circ} 40$, pp. 257-269

Mehdi Jabnoun. 2008. Adaptation des plantes au Stress salin, caractérisation des transporteurs de sodium et de potassium de la famille HKT chez le riz, Thèse de doctorat, Université de Montpellier p. 289.

Munns R, Testy M. 2008. Mechanism of salinity tolerance. Ann. Rev. Plant Biol., 59: 651-681.

Muhammad A, Muhammad Y A, Rachid A, Ejaz AW, Javed I, Muhammad M. 2010. Screening for salt tolerance in maize (Zea Mays L.) hybrids at an early seedling stage. Pak. J. Bot., 42(1): 141-154.

Munns R. 2002. Comparative physiology of salt and water stress. Plant, Cell Environ., 25: 239-250.

Nodichao L, Ake S, Jourdan C. 2007. Effet du potassium et du déficit hydrique modéré sur la transpiration et l'absorption potassique chez six génotypes de palmier au stade juvénile. Agronomie Africaine, 19(2): 151-160. DOI :

http://dx.doi.org/10.4314/aga.v19i2.171 9 
Nodichao L, Ake S, Jourdan C. 2008. Développement du système racinaire chez le palmier a huile selon l'origine génétique et le régime hydropotassique du sol. Agron. Afr., 20(3): $\quad 277-289 . \quad$ DOI : http://dx.doi.org/10.4314/aga.v20i3.462 64

Naidoo G, Mundree SG. 1992. Relationship between morphological and physiological responses to waterlogging and salinity in Sporobolus virginicus (L.) Kunth. Oecologia, 93: 360-366.

Ndiaye O, Diouf O, Adon B, Braconniar S.2007. Critères physiologiques discriminants au jeune âge pour la sélection de génotypes de palmier à huile (Elaeis guineensis Jacq.) tolérants à la sècheresse. Agronomie Africaine, 19(1): 1-12. DOI : http://dx.doi.org/10.4314/aga.v19i1.170 4

PFS.2014. Politique forestière du Sénégal 2005-2025, rapport final, 33-45.

Rashad. 2011. Influence of salt stress on growth and biochemical parameter of citrus rootstock, Pak. J. Bot., 43(4): 2135-2141.

Rafflegeau S. 2008. Dynamique d'implantation et conduite technique des plantations villageoises de palmier a huile au Cameroun : facteurs limitant et raisons des pratiques, $149 \mathrm{p}$.
Samira Ibn MH, Denden M, Bouthaina DM, Samia Ben M. 2011. Caractéristiques de la croissance et de la production en fruits chez trois variétés de piment (Capsicum annuum L.) sous stress salin. Tropicultura, 29(2): 75-81.

SEPAM. 2010. Programme d'eau potable et d'assainissement du millénaire. Plan local hydraulique et d'assainissementCommunauté rural de Niaguis/version finale. $54 \mathrm{p}$

Shaoib I. 2009-2013. ICBA Highlights and Achievements. International Center for Biosaline Agriculture: Dubai. United Arab Emirates; 40.

Serra H.2000. Effet of salinity on citrus, Ege University Faculty of Agriculture Department of Horticulture BornovaĐzmir/Turkey Anadolu. J. AARI., 10(1) 52-72.

Thouraya R, Imen I, Riadh I, Ahlem B, Hager J. 2013. Effet du stress salin sur le comportement physiologique et métabolique de trois variétés de piment (Capsicum annuum L.). Journal of Applied Biosciences, 66: 5060-5069. DOI :

http://dx.doi.org./10.4314/jab.v66i0.95 004 